\title{
Why are some brightest cluster galaxies forming stars?
}

\author{
Christopher P. O'Dea ${ }^{1}$, Alice Quillen ${ }^{2}$, Nicholas Zufelt ${ }^{2}$, Jaehong \\ Park $^{2}$, Alastair Edge ${ }^{3}$, Helen Russell ${ }^{4}$, Andy Fabian ${ }^{4}$, Stefi Baum \\ and The Spitzer BCG Project \\ ${ }^{1}$ Department of Physics, Rochester Institute of Technology, Rochester, NY 14623, USA \\ email: odea@cis.rit.edu \\ ${ }^{2}$ Department of Physics and Astronomy, University of Rochester, Rochester, NY 14627, USA \\ email: aquillen@pas.rochester.edu \\ ${ }^{3}$ Institute for Computational Cosmology, Department of Physics, Durham University, \\ Durham DH1 3LE, UK \\ email: alastair.edge@durham.ac.uk \\ ${ }^{4}$ Institute of Astronomy, Madingley Rd., Cambridge, CB3 0HA, UK \\ email: hrr27@ast.cam.ac.uk, acf@ast.cam.ac.uk \\ ${ }^{5}$ Center for Imaging Science, Rochester Institute of Technology, Rochester, NY 14623, USA \\ email: baum@cis.rit.edu
}

\begin{abstract}
We present first results from an imaging survey with the Spitzer Space Telescope of 62 brightest cluster galaxies with optical line emission located in the cores of X-ray luminous clusters selected from the ROSAT All-Sky Survey. We find that $1 / 3$ of these sources have signs of excess infrared emission; 22 objects of 62 are detected at $70 \mu \mathrm{m}$ and 19 have 8 to $5.8 \mu \mathrm{m}$ flux ratios above 0.98 . The strength of the excess emission correlates with the luminosity of the optical emission lines. Excluding the four systems dominated by an AGN, the excess mid-infrared emission in the remaining brightest cluster galaxies is likely powered by star formation. We find a correlation between mass deposition rate from a cooling flow model for the X-ray emission and the star formation rate estimated from the infrared luminosity. The star formation rates are $1 / 10$ to $1 / 100$ of the mass deposition rates expected in the absence of heating suggesting that the re-heating of the ICM is generally very effective in reducing the amount of mass cooling from the hot phase.
\end{abstract}

Keywords. stars: formation - galaxies: clusters: general - galaxies: active - galaxies: elliptical and lenticular, $\mathrm{cD}$ - (galaxies:) cooling flows - infrared: galaxies

\section{Introduction}

X-ray imaging of galaxy clusters show a density increase and a temperature decrease toward the centers of these systems, implying that gas should be cooling at rates of a few to $1000 M_{\odot} \mathrm{yr}^{-1}$ (e.g., Allen 2000). For a review of the era before the launch of the Chandra and XMM-Newton telescopes see Fabian (1994). A number of studies have found evidence for cooled gas and star formation, but at a rate below that corresponding to the cooling rate predicted from the X-ray observations (e.g., McNamara \& O'Connell 1989, Cardiel et al. 1998, Crawford et al. 1999). Cool plasma at half to one-third of ambient value has been detected but there seems to be a floor of gas temperatures below which cooling does not occur Peterson \& Fabian 2006. The resulting estimated or true cooling rates are about an order of magnitude lower than those derived from the Xray observations. Recent studies have focused on the nature of energy input into the Intracluster medium (ICM) which can balance the radiative energy losses. In particular 


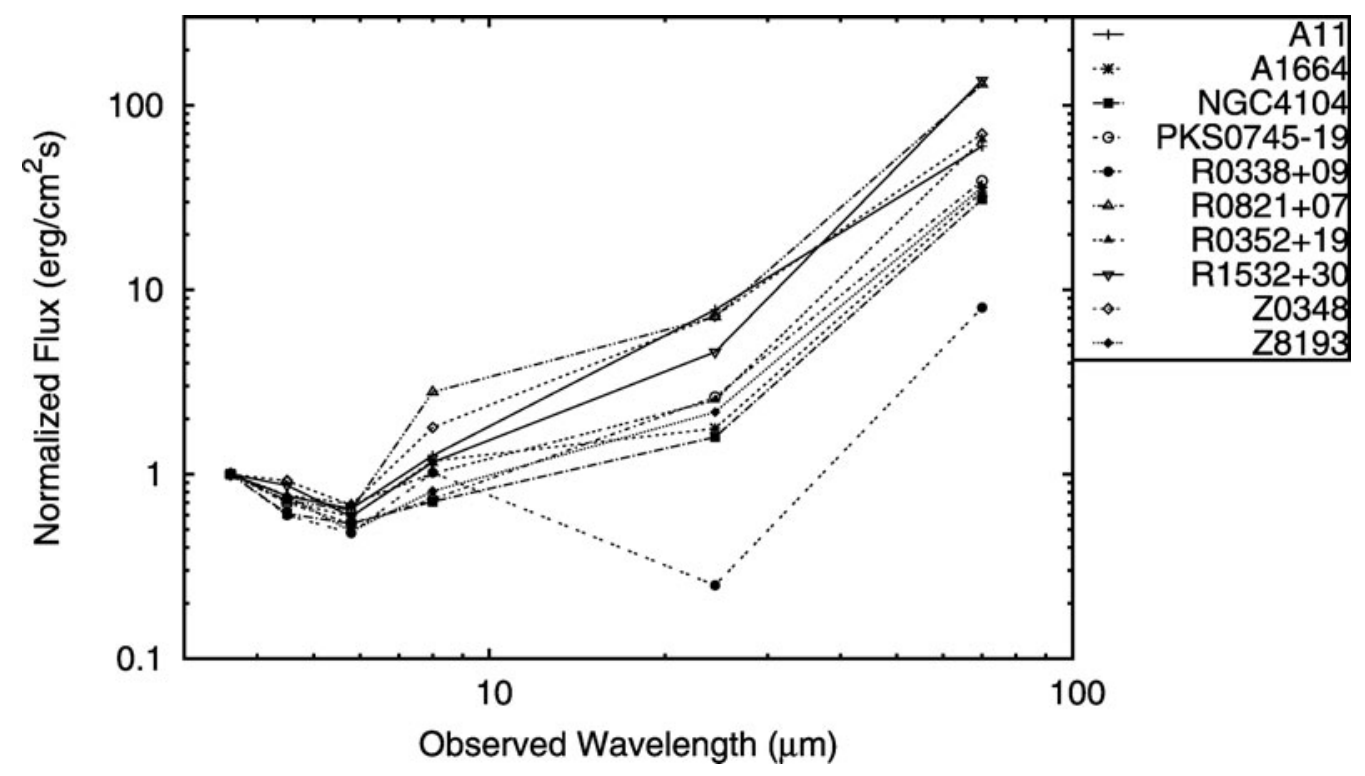

Figure 1. Infrared SED for for the BCGs with $F_{8 \mu \mathrm{m}} / F_{5.8 \mu \mathrm{m}}>1.3$. These galaxies have strong mid-infrared excesses compared to a quiescent elliptical galaxy. All were detected at $70 \mu \mathrm{m}$.

forms of AGN feedback could be effective by injecting energy into the ICM via giant buoyant bubbles, turbulence and cosmic rays, though conduction could also play a role in regulating the temperature. In recent years AGN outbursts have emerged as the best candidate energy source able to meet the energy requirements (e.g., Birzan et al. 2004, Dunn \& Fabian 2006, Rafferty et al. 2006).

Some form of feedback/heating seems necessary to explain the luminosity function of galaxies (e.g., Benson et al. 2003, Binney 2004) and the lack of gas at temperatures below a few keV in cool core clusters Peterson \& Fabian 2006. Star formation is likely to be the ultimate sink for any cooling gas in the cool core clusters and thus provides a constraint on the ability of heating to stop catastrophic cooling in the ICM. Thus, we undertook a census of star formation in Brightest Cluster Galaxies (BCGs) using Spitzer.

\section{Results}

The Spitzer Observations: We selected a flux limited sample of 62 BCGs based on cluster X-ray flux (Ebeling et al. 1998, Ebeling et al. 2000, Bohringer et al. 2004) and BCG H $\alpha$ flux Crawford et al. 1999. These criteria favor BCGs in cool core clusters. We obtained IRAC imaging in the 3.6, 4.5, 5.8, and 8 micron bands, and MIPS imaging in the 24 and 70 micron bands. The details of the Spitzer observations and reduction are given by Zufelt et al. 2007 and the results are discussed by O'Dea et al. 2007 .

The Infrared Excess: We find that in about one-third of the BCGS, there is an infrared excess above that expected for the passive old stellar population. 22 objects of 62 are detected at $70 \mu \mathrm{m}$ and 19 have 8 to $5.8 \mu \mathrm{m}$ flux ratios above 0.98 . Figure 1 shows the infrared SED for a selected set of BCGs showing the IR excess.

The BCGs in Abell 1068, Abell 2146, Zw 2089, and R0821+07 appear to be dominated by an AGN - they have warmer dust temperatures and high $[\mathrm{OIII}] / \mathrm{H} \beta$ ratios. The rest of the BCGs with the IR excess do not show evidence for optically luminous AGN (Zufelt et al. 2007, O'Dea et al. 2007) and thus appear to be dominated by star formation. 


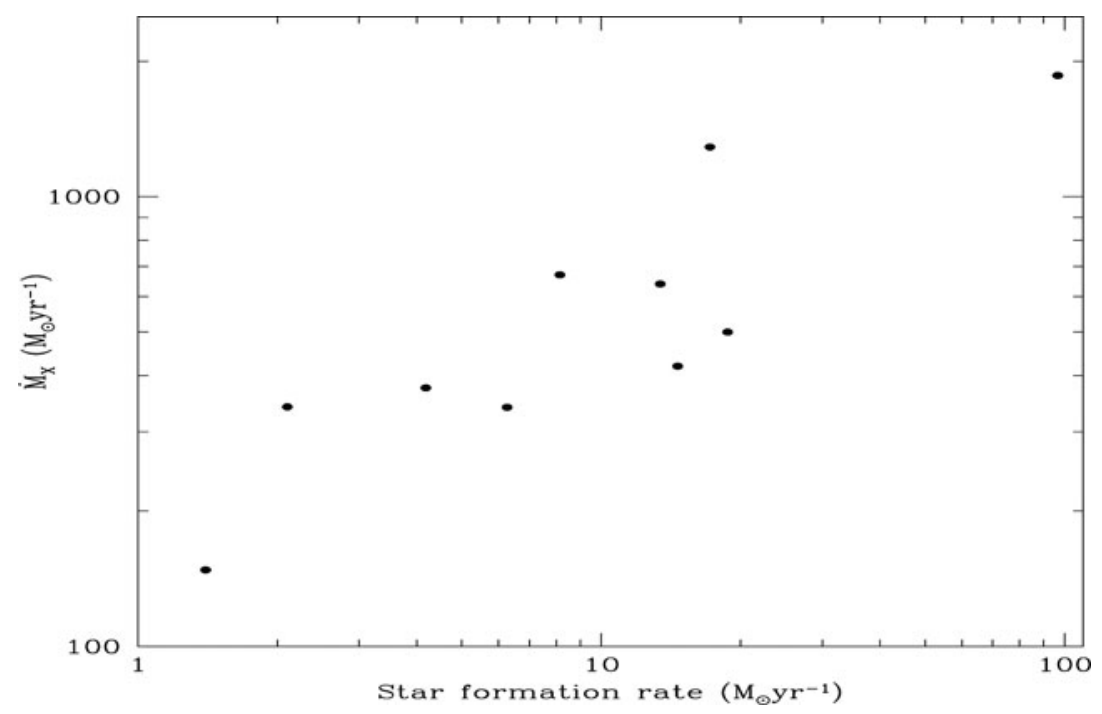

Figure 2. Mass deposition rates estimated from the X-ray emission $\left(\dot{M}_{X}\right)$ vs star formation rate estimated from the infrared luminosity for the sources with an IR excess.

The estimated IR luminosities, following Elbaz et al. (2002), are in the range $10^{43}$ to a few $\times 10^{45} \mathrm{ergs} / \mathrm{s}$. There are 9 BCGs (of which 4 are AGN dominated) which have luminosities $L_{I R}>10^{11} \mathrm{M}_{\odot}$ and are thus Luminous Infrared Galaxies (LIRGS).

Star Formation Rates: We estimated the star formation rates (SFR) using equation 5 by Bell (2003);

$$
\psi\left(M_{\odot} \mathrm{yr}^{-1}\right)=A\left(\frac{L_{I R}}{L_{\odot}}\right)\left(1+\sqrt{10^{9} L_{\odot} / L_{I R}}\right) .
$$

Here the constant $A=1.57 \times 10^{-10}$ for $L_{I R}>10^{11} L_{\odot}$ otherwise $A=1.17 \times 10^{-10}$. The SFR are in the range of about 1 to about $50 M_{\odot} /$ yr. The objects with SFR above about $50 M_{\odot} / \mathrm{yr}$ are the AGN dominated objects.

Mass deposition rates vs. SFR: Chandra and XMM-Newton archive data were processed and cleaned using the latest software and calibration files. X-ray spectra were extracted for the central $100 \mathrm{kpc}$ region of each cluster and fitted in XSPEC v11.3.2 with a single-temperature MEKAL model and a photoelectric absorption model. The best-fitting temperature value and model luminosity, for the energy range $0.01-50 \mathrm{keV}$, were used to calculate a measure of the radiative cooling rate in the absence of heating:

$$
\dot{M}_{X}=\left(\frac{2 \mu m_{H}}{5 k_{B}}\right) \frac{L(R<100 \mathrm{kpc})}{T(R<100 \mathrm{kpc})}
$$

where $\mu$ is the mean molecular weight. Studies of nearby clusters suggest that the real cooling rate of the gas is about one tenth of this value (e.g., Rafferty et al. 2006), so we can use $\dot{M}_{X}$ as a proxy for the mass deposition rate.

We have plotted mass deposition rates $\left(\dot{M}_{X}\right)$ against star formation rates estimated from the infrared luminosity in Figure 3. We find a correlation between the two quantities which is much stronger than between the X-ray and infrared luminosities. This suggests that the star formation rate is related to the predicted mass deposition rate in the X-ray gas. We estimate that the star formation rate ranges from 1/10 (at the lower end) to $1 / 100$ (at the higher end) of the predicted mass deposition rate. 


\subsection{Implications}

The observed correlation of $\dot{M}_{X}$ with SFR is consistent with the hypothesis that the cooling ICM is the source of the gas which is forming stars. This suggests that the concentration or compactness of the ICM is important in setting the star formation rate. However, the SFR is roughly a few percent of $\dot{M}_{X}$. This is consistent with the results from X-ray spectroscopy (e.g., Peterson \& Fabian 2006) which suggests that most of the gas with short cooling time at a few keV does not cool further. This fraction is a mean number and likely to be biased as our sample was chosen from objects bright in $\mathrm{H} \alpha$. Nevertheless this number could provide a constraint on the efficiency of feedback models that prevent the bulk of the ICM from cooling. If star formation is the ultimate sink for the cooling gas, then the fraction of the few $\mathrm{keV}$ gas which does cool all the way down should be comparable to the ratio SFR/ $\dot{M}_{X}-$ which we find to be roughly a few percent. This suggests that the re-heating mechanism (whatever it is) is very effective over a range of size scales and time scales.

\section{Acknowledgements}

This work is based in part on observations made with the Spitzer Space Telescope, which is operated by the Jet Propulsion Laboratory, California Institute of Technology under a contract with NASA. Support for this work at University of Rochester and Rochester Institute of Technology was provided by NASA through an award issued by JPL/Caltech.

\section{References}

Allen, S. W. 2000, MNRAS, 315, 269

Bell, E. F. 2003, ApJ, 586, 794

Benson, A. J., Bower, R. G., Frenk, C. S., et al. 2003, ApJ, 599, 38

Binney, J., 2004, MNRAS, 347, 1093

Birzan, L., Rafferty, D. A., McNamara, B. R., et al. 2004, ApJ, 607, 800

Bohringer, H., et al. 2004, A\&A, 425, 367

Cardiel, N., Gorgas, J., \& Aragon-Salamanca, A. 1998, Ap\&SS, 263, 83

Crawford, C. S., Allen, S. W., Ebeling, H., et al. 1999, MNRAS, 306, 857

Dunn, R. J. H. \& Fabian, A. C. 2006, MNRAS, 373, 959

Ebeling, H., Edge, A. C., Bohringer, H., et al. 1998, MNRAS, 301, 881

Ebeling, H., Edge, A. C., Allen, S. W., et al. 2000, MNRAS, 318, 333

Elbaz, D., Cesarsky, C. J., Chanial, P., et al. 2002, A\&A, 384, 848

Fabian, A. C. 1994, ARA\&A 32, 277

McNamara, B. R. \& O'Connell, R. W. 1989, AJ, 98, 2018

O'Dea, et al., 2007, in preparation

Peterson, J. R. \& Fabian, A. C., 2006, Physics Reports, 427, 1

Rafferty, D. A., McNamara, B. R., Nulsen, P. E. J., \& Wise, M. W. 2006, ApJ, 652, 216

Zufelt, N., et al. 2007, ApJS, in preparation. 